\title{
PACKING AND COVERING CONSTANTS FOR CERTAIN FAMILIES OF TREES. II(')
}

BY

\author{
A. MEIR AND J. W. MOON
}

\begin{abstract}
In an earlier paper we considered the problem of determining the packing and covering constants for families of trees whose generating function $y$ satisfied a relation $y=x \phi(y)$ for some power series $\phi$ in $y$. In the present paper we consider the problem for some families of trees whose generating functions satisfy a more complicated relation.
\end{abstract}

1. Introduction. Let $T_{n}$ denote a tree with $n$ nodes. The distance $d(u, v)$ between nodes $u$ and $v$ of $T_{n}$ is the number of edges in the unique path joining $u$ and $v$. (For definitions not given here see [1] or [6].)

A subset $\mathscr{P}$ of nodes of $T_{n}$ is a $k$-packing, where $k$ is a fixed positive integer, if $d(u, v)>k$ for every pair of distinct nodes $u$ and $v$ in $\mathcal{P}$. A subset $\mathcal{C}$ of nodes of $T_{n}$ is a $k$-covering if for every node $u$ of $T_{n}$ there exists a node $v$ of $C$ such that $d(u, v)<k$. The $k$-packing number $P_{k}\left(T_{n}\right)$ is the number of nodes in any largest $k$-packing of $T_{n}$, and the $k$-covering number $C_{k}\left(T_{n}\right)$ is the number of nodes in any smallest $k$-covering of $T_{n}$.

If $\mathscr{F}$ denotes a given family of trees, let $p_{k}(n)$ and $c_{k}(n)$ denote the average value of $P_{k}\left(T_{n}\right)$ and $C_{k}\left(T_{n}\right)$ over all trees $T_{n}$ in $\mathcal{F}$ that have $n$ nodes. In [5] (see also [2] and [3]) we determined the asymptotic behaviour of $p_{k}(n)$ and $c_{k}(n)$, as $n \rightarrow \infty$, for families of rooted trees whose generating functions $y=y(x)$ satisfied a functional relation of the type $y=x \phi(y)$, subject to some rather mild conditions. Our object here is to consider the usually more difficult case when the generating function does not satisfy a relation of this type.

In particular, we shall investigate rooted unlabelled trees and unrooted unlabelled trees. We shall show that the packing and covering constants

$$
p_{1}=\lim _{n \rightarrow \infty} p_{1}(n) / n \text { and } c_{1}=\lim _{n \rightarrow \infty} c_{1}(n) / n
$$

exist in these cases, and we shall obtain estimates for their values. It turns out that $p_{1}$ and $c_{1}$ have the same values for the family of unrooted unlabelled

Received by the editors March 4, 1976.

AMS (MOS) subject classifications (1970). Primary $05 C 05$.

Key words and phrases. Trees, packing constants, covering constants.

(')The preparation of this paper was assisted by grants from the National Research Council of Canada.

- American Mathematical Society 1977 
trees as they do for the family of rooted unlabelled trees.

2. Definitions and observations. Let $y_{n}$ denote the number of trees $T_{n}$ in $\mathscr{F}$ that have $n$ nodes. We assume that $y_{1}=1$ and that any nontrivial tree in $\mathscr{F}$ may be constructed by joining the roots of a collection of smaller trees from $F$ to a new node that serves as the root of the resulting tree. We further assume that this construction gives rise to a recurrence relation that expresses $y_{n}$ in terms of $y_{1}, \cdots, y_{n-1}$ for $n \geqslant 2$. Finally, we assume that there exists an operator $\gamma\left\{h_{1}(x), h_{2}(x), \cdots\right\}$ defined for any (possibly infinite) sequence of power series $h_{1}(x), h_{2}(x), \ldots$, such that if

$$
y=y(x)=\sum_{1}^{\infty} y_{n} x^{n},
$$

then the recurrence relation for $y_{n}$ can be expressed in terms of generating functions as

$$
y(x)=\gamma\left\{y(x), y\left(x^{2}\right), \cdots\right\}
$$

For notational convenience we shall write this simply as

$$
y(x)=\Gamma\{y(x)\}
$$

More generally, if $w(x, z)$ is any power series in the two variables $x$ and $z$, then we let

$$
\Gamma\{w(x, z)\}=\gamma\left\{w(x, z), w\left(x^{2}, z^{2}\right), \cdots\right\}
$$

where $w(x, z), w\left(x^{2}, z^{2}\right), \cdots \quad$ are interpreted as functions of $x$ for fixed values of $z$.

The results in [5] can be used to determine recursively the number of trees $T_{n}$ in $\mathscr{F}$ for which $P_{k}\left(T_{n}\right)=i$ or for which $C_{k}\left(T_{n}\right)=i$, for $1<i<n$. However, because of the general difficulty in establishing the existence and finding the explicit values of the packing and covering constants

$$
p_{k}=\lim _{n \rightarrow \infty} p_{k}(n) / n \text { and } c_{k}=\lim _{n \rightarrow \infty} c_{k}(n) / n
$$

when the operator $\Gamma$ cannot be expressed as $\Gamma\{y(x)\}=x \phi(y(x))$, we shall restrict our attention to the constants $p_{1}$ and $c_{1}$. We shall use the fact (see [4]) that $P_{2 k}\left(T_{n}\right)=C_{k}\left(T_{n}\right)$ for any tree $T_{n}$ in determining $c_{1}$, so we shall actually be considering 1-packings and 2-packings.

Let $p_{n i}$ and $c_{n i}$ denote the fraction of trees $T_{n}$ in $\mathscr{F}$ for which $P_{1}\left(T_{n}\right)=i$ and $P_{2}\left(T_{n}\right)=C_{1}\left(T_{n}\right)=i$, respectively, for $1 \leqslant i \leqslant n$. If

$$
P=P(x, z)=\sum_{n, i} p_{n i} y_{n} z^{i} x^{n}
$$

and

$$
C=C(x, z)=\sum_{n, i} c_{n i} y_{n} z^{i} x^{n}
$$


then clearly $P(x, 1)=C(x, 1)=y(x)$. Furthermore, if

$$
p(x)=\sum_{n} p_{1}(n) y_{n} x^{n} \text { and } c(x)=\sum_{n} c_{1}(n) y_{n} x^{n},
$$

where $p_{1}(n)$ and $c_{1}(n)$ denote the expected packing and covering numbers defined in $\$ 1$, then

$$
p(x)=(\partial / \partial z)(P(x, z))_{z=1}
$$

and

$$
c(x)=(\partial / \partial z)(C(x, z))_{z=1^{\circ}}
$$

Thus if we can determine the generating functions $P(x, z)$ and $C(x, z)$ then, in principle, we can determine the expected packing and covering numbers $p_{1}(n)$ and $c_{1}(n)$. In order to obtain defining relations for $P(x, z)$ and $C(x, z)$, we need to introduce some auxiliary functions.

3. Preliminary results. We say that a rooted tree $T_{n}$ with root $r$ is a type $(k, j)$ tree, where $0 \leqslant j<k$, if

$$
\max \{d(r, \mathscr{P}): \mathscr{P} \text { is a largest } k \text {-packing of } T\}=j \text {. }
$$

Thus, for example, a type $(k, 0)$ tree is one in which the root belongs to every largest $k$-packing. Let the generating functions $F_{0}=F_{0}(x, z)$ and $F_{1}=$ $F_{1}(x, z)$ be defined with respect to the type $(1,0)$ and type $(1,1)$ trees in $\mathscr{F}$ in the same way that $P(x, y)$ was defined with respect to all the trees in $\mathscr{F}$; thus, for example, the coefficient of $z^{i} x^{n}$ in $F_{0}$ is the number of type $(1,0)$ trees $T_{n}$ in $\mathscr{F}$ for which $P_{1}\left(T_{n}\right)=i$. And let the generating functions $G_{0}=G_{0}(x, z), G_{1}$ $=G_{1}(x, z)$, and $G_{2}=G_{2}(x, z)$ be similarly defined with respect to the trees of type $(2,0),(2,1)$, and $(2,2)$ in $\mathscr{F}$. Then,

$$
F_{0}+F_{1}=P
$$

and

$$
G_{0}+G_{1}+G_{2}=C \text {. }
$$

Furthermore, if $f_{i}=f_{i}(x)=F_{i}(x, 1)$ and $g_{j}=g_{j}(x)=G_{j}(x, 1)$, then

$$
f_{0}(x)+f_{1}(x)=g_{0}(x)+g_{1}(x)+g_{2}(x)=y(x) \text {. }
$$

Trees of type $(k, j)$ were characterized in [5] and it was shown that the characterization yielded a defining relation for the generating functions $P$ and $C$ in terms of the defining relation for $y(x)$. In the present context these results may be expressed as follows when $k=1$ and $k=2$.

LEMMA 1. If $y=\Gamma\{y\}$, then

and

$$
F_{0}=z \Gamma\left\{F_{1}\right\}, \quad F_{1}=\Gamma\{P\}-\Gamma\left\{F_{1}\right\},
$$

$$
P=\Gamma\{P\}+(z-1) \Gamma\left\{F_{1}\right\} .
$$


LEMMA 2. If $y=\Gamma\{y\}$, then

$$
\begin{aligned}
& G_{0}=z \Gamma\left\{G_{2}\right\}, \\
& G_{1}=z \Gamma\left\{G_{0} z^{-1}+G_{1}+G_{2}\right\}-z \Gamma\left\{G_{1}+G_{2}\right\}, \\
& G_{2}=\Gamma\left\{G_{1}+G_{2}\right\}-\Gamma\left\{G_{2}\right\},
\end{aligned}
$$

and

$$
C=z \Gamma\left\{G_{0} z^{-1}+G_{1}+G_{2}\right\}+(1-z) G_{2} .
$$

Notice that (3.4) and (3.5) both reduce to the relation $y=\Gamma\{y\}$ when $z=1$. These lemmas and (2.2) and (2.3) imply the following relations for $p(x)$ and $c(x)$.

COROLLARY 1. If $y=\Gamma\{y\}$, then

$$
p(x)=(\partial / \partial z)(\Gamma\{P\})_{z=1}+f_{0}(x) .
$$

COROLLARY 2. If $y=\Gamma\{y\}$, then

$$
c(x)=(\partial / \partial z)\left(\Gamma\left\{G_{0} z^{-1}+G_{1}+G_{2}\right\}\right)_{z=1}+g_{0}(x)+g_{1}(x) .
$$

4. Rooted unlabelled trees. Let $\mathscr{F}$ denote the family of (nonisomorphic) rooted unlabelled trees. It is well known (see, e.g., [1, p. 52]) that the generating function $y(x)$ for trees in $\mathscr{F}$ satisfies the relation

$$
y(x)=x \exp \sum_{1}^{\infty} y\left(x^{k}\right) / k \text {. }
$$

Thus the family $\mathscr{F}$ satisfies (2.1) with

$$
\Gamma\{y(x)\}=x \exp \sum_{1}^{\infty} y\left(x^{k}\right) / k
$$

We observe, for later use, that if $w(x, z)$ denotes any power series in $x$ and $z$, then

$$
\frac{\partial}{\partial z} \Gamma\{w(x, z)\}=\Gamma\{w(x, z)\} \sum_{1}^{\infty} \frac{\partial w\left(x^{k}, z^{k}\right)}{\partial z} z^{k-1} .
$$

The following results are straightforward consequences of Lemmas 1 and 2, Corollaries 1 and 2, and relations (4.2) and (4.3).

THEOREM 1. If $\mathscr{F}$ denotes the family of rooted unlabelled trees then

$$
p(x)=y(x) \sum_{1}^{\infty} p\left(x^{k}\right)+f_{0}(x)
$$

where

$$
f_{0}(x)=y(x)-f_{1}(x)=x \exp \sum_{1}^{\infty} f_{1}\left(x^{k}\right) / k
$$


THEOREM 2. If $\mathcal{F}$ denotes the family of rooted unlabelled trees, then

$$
c(x)=y(x) \sum_{1}^{\infty}\left\{c\left(x^{k}\right)-g_{0}\left(x^{k}\right)\right\}+g_{0}(x)+g_{1}(x),
$$

where

$$
\begin{aligned}
& g_{0}(x)=x \exp \sum_{1}^{\infty} g_{2}\left(x^{k}\right) / k, \\
& g_{1}(x)=y(x)-g_{0}(x) \exp \sum_{1}^{\infty} g_{1}\left(x^{k}\right) / k,
\end{aligned}
$$

and

$$
g_{2}(x)=y(x)-g_{0}(x)-g_{1}(x) .
$$

We shall use the following lemma (see [5] for the proof of a similar result) in estimating the packing and covering constants for the family $\mathscr{F}$ of rooted unlabelled trees.

LEMMA 3. Let $A(x)=\sum_{0}^{\infty} a_{n} x^{n}, B(x)=\sum_{0}^{\infty} b_{n} x^{n}$, and $A(x) B(x)=\Sigma_{0}^{\infty} c_{n} x^{n}$ and suppose there exists a positive constant $\rho$ such that $B(\rho) \neq 0$ and $b_{n}=$ $O\left(n^{-3 / 2} \rho^{-n}\right)$ as $n \rightarrow \infty$.

(i) If $a_{n}=O\left(n^{-3 / 2} \rho^{-n}\right)$, then $c_{n}=O\left(n^{-3 / 2} \rho^{-n}\right)$ as $n \rightarrow \infty$.

(ii) If $a_{n} \sim c n^{-1 / 2} \rho^{-n}$, where $c>0$, then $c_{n} \sim B(\rho) a_{n}$ as $n \rightarrow \infty$.

Otter [7] (see also [1, pp. 209-213]) showed that the generating function $y(x)$ of the family $\mathscr{F}$ of rooted unlabelled trees has radius of convergence $\rho=.3383 \cdots$ and that about the point $x=\rho$ it has.an expansion of the type

$$
y(x)=1-b(\rho-x)^{1 / 2}+b_{2}(\rho-x)^{1}+b_{3}(\rho-x)^{3 / 2}+\ldots,
$$

where $b=2.6811 \cdots$. From this he deduced that

$$
y_{n} \sim(b / 2 \sqrt{\pi}) \rho^{-n+1 / 2} n^{-3 / 2}
$$

as $n \rightarrow \infty$.

We remark, for later use, that it follows from (4.1) that

$$
x y^{\prime}(x)(1-y(x))=y(x) \cdot Q(x)
$$

where

$$
Q(x)=1+\sum_{2}^{\infty} y^{\prime}\left(x^{k}\right) x^{k}
$$

relations (4.10) and (4.12) imply (see [1, pp. 212, 213]) that

$$
Q(\rho)=\frac{1}{2} b^{2} \rho=1.21599 \cdots .
$$




\section{The packing constant for rooted unlabelled trees.}

THEOREM 3. If $\mathcal{F}$ denotes the family of rooted, unlabelled trees, then there exists a constant $p_{1}$, where $.603<p_{1}<.609$, such that $p_{1}(n) / n \rightarrow p_{1}$ as $n \rightarrow \infty$.

PROOF. For notational convenience we shall let $C_{n}(f)$ denote the coefficient of $x^{n}$ in any power series $f(x)$.

Equation (4.4) can be rewritten as

$$
p(x)=(y /(1-y)) H(x)+f_{0}(x)
$$

where

$$
H(x)=\sum_{2}^{\infty} p\left(x^{k}\right)+f_{0}(x)
$$

and, in view of (4.12), this can be rewritten as

$$
p(x)=x y^{\prime}(x) H(x) / Q(x)+f_{0}(x) .
$$

Since $0 \leqslant e_{n}\left(f_{0}\right)<y_{n}$, equation (4.11) implies that $e_{n}\left(f_{0}\right)=$ $O\left(n^{-3 / 2} \rho^{-n}\right)$. It follows from (4.11) and the definition of $p(x)$ that $\Sigma_{2}^{\infty} p\left(x^{k}\right)$ has radius of convergence $\rho^{1 / 2}>\rho=.3383 \cdots$. Hence, $\mathcal{C}_{n}(H)=$ $O\left(n^{-3 / 2} \rho^{-n}\right)$.

Since $Q(x)$ is analytic for $|x|<\rho^{1 / 2}$, it follows from (4.14) that $Q(x) \neq 0$ for $|x|<\rho+2 \varepsilon$ for some $\varepsilon>0$; thus $1 / Q(x)$ is analytic for $|x|<\rho+2 \varepsilon$ and, consequently, $C_{n}(1 / Q)=O\left((\rho+\varepsilon)^{-n}\right)=O\left(n^{-3 / 2} \rho^{-n}\right)$. These facts imply that $\mathcal{C}_{n}(H / Q)=O\left(n^{-3 / 2} \rho^{-n}\right)$ by Lemma 3(i).

Moreover, $C_{n}\left(x y^{\prime}\right) \sim c n^{-1 / 2} \rho^{-n}$, where $c=b \rho^{1 / 2} / 2 \sqrt{\pi}$, by (4.11). Therefore, we may apply Lemma 3(ii) to (5.2), and conclude that

$$
p_{1}(n) y_{n} \sim n y_{n} H(\rho) / Q(\rho),
$$

or that

$$
p_{1}(n) / n \rightarrow p_{1}=H(\rho) / Q(\rho)
$$

as $n \rightarrow \infty$. Since $Q(\rho)$ is known, by (4.12), it remains to estimate $H(\rho)$.

Let

$$
f_{0}(x)=\sum_{1}^{\infty} \alpha_{n} x^{n} \text { and } f_{1}(x)=\sum_{1}^{\infty} \beta_{n} x^{n}
$$

Relations (4.1) and (4.5) imply that

$$
y(x)=x \prod_{1}^{\infty}\left(1-x^{j}\right)^{-y_{j}} \text { and } f_{0}(x)=x \prod_{1}^{\infty}\left(1-x^{j}\right)^{-\beta_{j}},
$$

by a standard argument (see, e.g., [1, p. 54]). These relations and the fact that $y=f_{0}+f_{1}$ may be combined to show that

$$
f_{0}(x) e^{f_{0}(x)}=x e^{y(x)} \prod_{1}^{\infty}\left\{\left(1-x^{j}\right) e^{x^{j}}\right\}^{-\beta_{j}}=y(x) \prod_{1}^{\infty}\left\{\left(1-x^{j}\right) e^{x^{j}}\right\}^{\alpha_{j}}
$$


We observe that $y(\rho)=1$, by (4.10), and that $\alpha_{1}=\alpha_{3}=1$. Since $(1-t) e^{t}<$ 1 for $0<t<1$, it follows that

$$
f_{0}(\rho) e^{f_{0}(\rho)}<(1-\rho)\left(1-\rho^{3}\right) e^{\rho+\rho^{3}}<.9275 .
$$

Furthermore, $\beta_{2}=\beta_{3}=1$, so

$$
f_{0}(\rho) e^{f_{0}(\rho)}>\rho e\left(1-\rho^{2}\right)^{-1}\left(1-\rho^{3}\right)^{-1} e^{-\rho^{2}-\rho^{3}}>.9268 .
$$

These inequalities imply that

$$
.540<f_{0}(\rho)<.541 .
$$

$$
\text { Now, } \begin{aligned}
p_{1}(1)= & p_{1}(2)=1, p_{1}(3)=2, \text { and } p_{1}(n)<n-1 \text { for } n \geqslant 2 \text {; hence, } \\
& x+x^{2}+4 x^{3}<p(x)<x y^{\prime}(x)-y(x)+x
\end{aligned}
$$

for $x>0$. The lower bound for $p(x)$ implies that

$$
\sum_{2}^{\infty} p\left(\rho^{k}\right)>\rho^{2}(1-\rho)^{-1}+\rho^{4}\left(1-\rho^{2}\right)^{-1}+4 \rho^{6}\left(1-\rho^{3}\right)^{-1}>.193 .
$$

And, since $y(x)>x+x^{2}+2 x^{3}$, the upper bound implies that

$$
\sum_{2}^{\infty} p\left(\rho^{k}\right)<Q(\rho)-1-\rho^{4}\left(1-\rho^{2}\right)^{-1}-2 \rho^{6}\left(1-\rho^{3}\right)^{-1}<.199,
$$

appealing to (4.14). These estimates and (5.3) yield the inequality

$$
.733<H(\rho)<.740 \text {. }
$$

When we combine this with (4.14) we find that

$$
.603<p_{1}=H(\rho) / Q(\rho)<.609 \text {, }
$$

as required.

We remark that it can be shown that approximately $35 \%$ of the trees $T_{n}$ in $\mathscr{F}$ are type $(1,0)$ trees and approximately $65 \%$ are type $(1,1)$ trees, when $n$ is large.

\section{The covering constant for rooted unlabelled trees.}

THEOREM 4. If $\mathcal{F}$ denotes the family of rooted unlabelled trees, then there exists a constant $c_{1}$, where $.332<c_{1}<.335$, such that $c_{1}(n) / n \rightarrow c$, as $n \rightarrow \infty$.

Proof. If we let

$$
R(x)=g_{1}(x)+\sum_{2}^{\infty}\left\{c\left(x^{k}\right)-g_{0}\left(x^{k}\right)\right\}
$$

and appeal to (4.12) and (4.13) again, we find that equation (4.6) can be rewritten as

$$
c(x)=x y^{\prime}(x) \cdot(R(x) / Q(x))+g_{0}(x)+g_{1}(x) .
$$

The same argument that led to (5.2) shows that $c_{1}(n) / n \rightarrow c_{1}=R(\rho) / Q(\rho)$, 
as $n \rightarrow \infty$, so it remains to estimate $R(\rho)$.

Since $c_{1}(1)=1$ and $1<c_{1}(n)<\frac{1}{2} n$ when $n>2$, it is not difficult to see that

$$
\begin{aligned}
x^{2}+2 x^{3} & <y(x)-g_{0}(x) \leqslant c(x)-g_{0}(x)<c(x)-x \\
& <x^{2}+2 x^{3}+\frac{1}{2} \sum_{4}^{\infty} n y_{n} x^{n}<\frac{1}{2} x y^{\prime}(x)-\frac{1}{2} x-x^{3}
\end{aligned}
$$

for $x>0$. From this we may conclude, by an argument similar to the one used in $\S 5$, that

$$
.0179<\sum_{2}^{\infty}\left\{c\left(\rho^{k}\right)-g_{0}\left(\rho^{k}\right)\right\}<.020 .
$$

We now turn to the problem of estimating $g_{1}(\rho)$.

We observe that

$$
\begin{aligned}
& \sum_{2}^{\infty}\left\{g_{1}\left(\rho^{k}\right)+g_{2}\left(\rho^{k}\right)\right\} / k=\sum_{2}^{\infty} y\left(\rho^{k}\right) / k-\sum_{2}^{\infty} g_{0}\left(\rho^{k}\right) / k \\
& <\ln (1 / \rho e)-\sum_{2}^{\infty} \rho^{k} / k=\ln (1 / \rho e)+\ln (1-\rho)+\rho<.0092,
\end{aligned}
$$

where we have appealed to (4.1) and the fact that $g_{0}(x)>x$. Since $g_{1}(x)>x^{2}$ $+x^{3}$, it follows that

$$
\sum_{2}^{\infty} g_{1}\left(\rho^{k}\right) / k>-\ln \left(1-\rho^{2}\right)-\ln \left(1-\rho^{3}\right)-\rho^{2}-\rho^{3}>.0078
$$

Equations (4.7)-(4.9) imply that

$$
g_{0}(x)=\left(y(x)-g_{1}(x)\right) \exp \left\{-\sum_{1}^{\infty} g_{1}\left(x^{k}\right) / k\right\}
$$

and

$$
y(x)-g_{1}(x)=x \exp \left(y(x)-g_{0}(x)+\sum_{2}^{\infty}\left\{g_{1}\left(x^{k}\right)+g_{2}\left(x^{k}\right)\right\} / k\right) .
$$

If we let $z=1-g_{1}(\rho)$ and apply inequalities (6.3) and (6.4) to (6.5) we find that

$$
z e^{z-1.0092}<g_{0}(\rho)<z e^{z-1.0078}
$$

When we apply (6.3) and (6.4) to (6.6) we obtain the inequality

$$
\rho e^{1.0078}<z e^{8_{0}(\rho)}<\rho e^{1.0092} .
$$

These last two inequalities imply that $.6130<z<.6140$, or that

$$
.3860<g_{1}(\rho)<.3870 \text {. }
$$


This last inequality combined with (4.14) and (6.2) yields the estimate

$$
.332<c_{1}=R(\rho) / Q(\rho)<.335 \text {, }
$$

as required.

We remark that it can be shown that approximately $15 \%, 37 \%$, and $48 \%$ of the trees $T_{n}$ in $\mathscr{F}$ are of type $(2,0),(2,1)$, and $(2,2)$, respectively, when $n$ is large.

7. Unrooted unlabelled trees. Let $\mathcal{G}$ denote the family of (nonisomorphic) unrooted unlabelled trees. In what follows, functions such as $P(x, z), C(x, z), y(x)$, etc. will denote the same functions as were defined earlier with respect to the family $\mathcal{F}$ of rooted unlabelled trees; functions such as $\bar{P}(x, z), \bar{C}(x, z), \bar{y}(x)$, etc. will denote the corresponding functions defined with respect to the family $\mathcal{G}$.

Otter [7] (see also [1, p. 57]) has shown that $\bar{y}(x)$ can be expressed in terms of $y(x)$ by the relation

$$
\bar{y}(x)=y(x)+\frac{1}{2}\left(y^{2}(x)-y\left(x^{2}\right)\right)
$$

and from this and (4.10) he deduced that

$$
\bar{y}_{n} \sim\left(b^{3} / 4 \sqrt{\pi}\right) \rho^{-n+3 / 2} n^{-5 / 2}
$$

as $n \rightarrow \infty$, when $\bar{y}_{n}$ denotes the number of unrooted unlabelled trees with $n$ nodes, and $b$ and $\rho$ are the constants defined in \$4. It turns out that the generating functions $\bar{p}(x)$ and $\bar{c}(x)$ for the expected packing and covering numbers of trees in $\mathcal{G}$ can also be expressed in terms of the corresponding generating functions $p(x)$ and $c(x)$ for trees in the family $\mathscr{F}$.

THEOREM 5. If $\mathcal{G}$ denotes the family of unrooted unlabelled trees, then

$$
\bar{p}(x)=p(x)(1-y(x))+p\left(x^{2}\right)+\frac{1}{2}\left(f_{0}^{2}(x)-f_{0}\left(x^{2}\right)\right),
$$

and

$$
\begin{aligned}
\bar{c}(x)= & c(x)(1-y(x))+c\left(x^{2}\right) \\
& +\frac{1}{2}\left(g_{0}^{2}(x)-g_{0}\left(x^{2}\right)+2 g_{0}(x) g_{1}(x)\right) .
\end{aligned}
$$

Proof. Otter's dissimilarity characteristic theorem (see [7] or [1, p. 57]) states, in effect, that the number of unrooted unlabelled trees with $n$ nodes equals the number of rooted unlabelled trees with $n$ nodes minus the number of trees with $n$ nodes that can be formed by joining the roots of two smaller nonisomorphic rooted unlabelled trees. If $D$ denotes the tree formed by joining the roots of two rooted trees $A$ and $B$, then it is not difficult to see that $p_{1}(D)=p_{1}(A)+p_{1}(B)$ unless $A$ and $B$ are both type $(1,0)$ trees in which case $p_{1}(D)=p_{1}(A)+p_{1}(B)-1$. 
If we express Otter's dissimilarity characteristic theorem in terms of generating functions we find that

$$
\begin{aligned}
\bar{P}(x, z)=P(x, z)-\frac{1}{2}\{ & \left(P^{2}(x, z)-P\left(x^{2}, z^{2}\right)\right) \\
- & \left.\left(1-z^{-1}\right)\left(F_{0}^{2}(x, z)-F_{0}\left(x^{2}, z^{2}\right)\right)\right\} .
\end{aligned}
$$

The factor $\frac{1}{2}$ is present because the order of the subtrees $A$ and $B$ is immaterial; the term involving $\left(1-z^{-1}\right)$ corrects the exponent of $z$ in the exceptional case when both $A$ and $B$ are type $(1,0)$ trees; and the terms $-P\left(x^{2}, z^{2}\right)$ and $-F_{0}\left(x^{2}, z^{2}\right)$ exclude the possibility that $A=B$. (Notice that (7.5) reduces to (7.1) when $z=1$.) Equation (7.3) follows upon differentiating both sides of equation (7.5) with respect to $z$ and then setting $z=1$.

The derivation of (7.4) is quite similar. The main difference is that $c_{1}(D)=$ $c_{1}(A)+c_{1}(B)$ unless $A$ and $B$ are both type $(2,0)$ trees, or one of them is a type $(2,0)$ tree and the other is a type $(2,1)$ tree; in these two cases $c_{1}(D)=c_{1}(A)+c_{1}(B)-1$. (Notice that the latter case automatically excludes the possibility that $A=B$.) It is not difficult to see that this implies that

$$
\begin{aligned}
\bar{C}(x, z)=C(x, z)-\frac{1}{2}\{ & \left(C^{2}(x, z)-C\left(x^{2}, z^{2}\right)\right)-\left(1-z^{-1}\right) \\
& \left.\cdot\left(G_{0}^{2}(x, z)-G_{0}\left(x^{2}, z^{2}\right)+2 G_{0}(x, z) G_{1}(x, z)\right)\right\} .
\end{aligned}
$$

Equation (7.4) now follows upon differentiating both sides of this equation with respect to $z$ and then setting $z=1$.

THEOREM 6. If $\mathcal{G}$ denotes the family of unrooted unlabelled trees, then $\bar{p}_{1}(n) / n \rightarrow p_{1}$ and $\bar{c}_{1}(n) / n \rightarrow c_{1}$, when $p_{1}$ and $c_{1}$ denote the packing and covering constants of the family $\mathscr{F}$ of rooted unlabelled trees.

Proof. For notational convenience we shall let $\phi_{1}, \phi_{2}, \cdots$ denote power series in which the coefficient of $x^{n}$ can be shown to be $O\left(\rho^{-n} n^{-3 / 2}\right)$.

Equations (7.3) and (5.1) imply that

$$
\begin{aligned}
\bar{p}(x)= & y(x)\left(H(x)-f_{0}(x)\right)+f_{0}(x)+\frac{1}{2} f_{0}^{2}(x) \\
& +p\left(x^{2}\right)-\frac{1}{2} f_{0}\left(x^{2}\right) .
\end{aligned}
$$

It follows from (4.5) that

$$
f_{0}^{\prime}=f_{0}\left(y^{\prime}-f_{0}^{\prime}\right)+\phi_{1} .
$$

If we differentiate both sides of equation (7.6) and apply this relation for $f_{0}^{\prime}$, we find that

$$
\begin{aligned}
\bar{p}^{\prime} & =y^{\prime}\left(H-f_{0}\right)+f_{0}^{\prime}+f_{0} f_{0}^{\prime}+\phi_{2} \\
& =y^{\prime}\left(H-f_{0}\right)+f_{0}\left(y^{\prime}-f_{0}^{\prime}\right)+f_{0} f_{0}^{\prime}+\phi_{3}=y^{\prime} H+\phi_{3} .
\end{aligned}
$$


It follows from Lemma 3(ii), therefore, that

$$
n \bar{p}_{1}(n) \bar{y}_{n} \sim n y_{n} H(\rho),
$$

or that

$$
\bar{p}_{1}(n) / n \sim y_{n} H(\rho) / n \bar{y}_{n} \sim 2 H(\rho) / b^{2} \rho=H(\rho) / Q(\rho)=p_{1},
$$

as $n \rightarrow \infty$, by (7.2), (4.11), (4.14), and Theorem 3. This completes the proof of the first part of the theorem.

Equations (7.4), (4.12), and (6.1) imply that

$$
\begin{aligned}
\bar{c}(x)= & y(x) R(x)+(1-y(x))\left(g_{0}(x)+g_{1}(x)\right) \\
& +\frac{1}{2} g_{0}^{2}(x)+g_{0}(x) \cdot g_{1}(x)+c\left(x^{2}\right)-\frac{1}{2} g_{0}\left(x^{2}\right)
\end{aligned}
$$

When we differentiate this and use the fact that $y=g_{0}+g_{1}+g_{2}$, we obtain

$$
\bar{c}^{\prime}=y^{\prime} R+y^{\prime}(1-y)+g_{1}^{\prime}\left(y-g_{1}\right)-g_{2}^{\prime}\left(1-g_{2}\right)+\phi_{4} \text {. }
$$

From (4.12) and (4.13) we conclude that $y^{\prime}(1-y)=\phi_{5}$; hence,

$$
\bar{c}^{\prime}=y^{\prime} R+g_{1}^{\prime}\left(y-g_{1}\right)-g_{2}^{\prime}\left(1-g_{2}\right)+\phi_{6} .
$$

It follows from equations (4.7) and (4.8) and Lemma 3(i) that

$$
g_{0}^{\prime}=g_{0} g_{2}^{\prime}+\phi_{7} \text { and } y^{\prime}-g_{1}^{\prime}=\left(y-g_{1}\right)\left(g_{1}^{\prime}+g_{2}^{\prime}\right)+\phi_{8}
$$

These two equations and the fact that $y=g_{0}+g_{1}+g_{2}$ imply that

$$
g_{1}^{\prime}\left(y-g_{1}\right)=g_{2}^{\prime}\left(1-g_{2}\right)+\phi_{9} \text {. }
$$

Consequently,

$$
\bar{c}^{\prime}=y^{\prime} R+\phi_{10}
$$

by (7.7) and (7.8). Hence,

$$
n \bar{c}_{1}(n) \bar{y}_{n} \sim n y_{n} R(\rho), \text { or } \bar{c}_{1}(n) / n \rightarrow c_{1}=R(\rho) / Q(\rho)
$$

as $n \rightarrow \infty$, by the same argument as before.

We remark that it is not true that $p_{1}(n)=\bar{p}_{1}(n)$ and $c_{1}(n)=\bar{c}_{1}(n)$ for all $n$. For example, $p_{1}(5)=29 / 9, \bar{p}_{1}(5)=10 / 3, c_{1}(5)=16 / 9$ and $\bar{c}_{1}(5)=5 / 3$.

\section{REFERENCES}

1. F. Harary and E. Palmer, Graphical enumeration, Academic Press, New York, 1973. MR 50 \#9682.

2. A. Meir and J. W. Moon, The expected node-independence number of random trees, Nederl. Akad. Wetensch. Proc. Ser. A 76 = Indag. Math. 35 (1973), 335-341. MR 49 \# 10607.

3. in Graph Theory, Academia Praha, 1975, pp. 351-363. 
4. __ Relations between packing and covering numbers of a tree, Pacific J. Math. 61 (1975), 225-233.

5. __ Packing and covering constants for certain families of trees. I, J. Graph Theory (to appear).

6. J. W. Moon, Counting labelled trees, Canad. Math. Monos., No. 1, Canad. Math. Congress, Montreal, Que., 1970. MR 43 \#98.

7. R. Otter, The number of trees, Ann. of Math. (2) 49 (1948), 583-599. MR 10, 53.

Departmant of Mathematics, University of Alberta, Edmonton, Alberta, Canada 